Published in final edited form as:

Curr Neurol Neurosci Rep. 2012 October ; 12(5): 618-627. doi:10.1007/s11910-012-0294-3.

\title{
Cognitive Impairment in Multiple Sclerosis
}

\author{
Jesus Lovera, MD and \\ Department of Neurology Louisiana State University Health Sciences Center-New Orleans 1542 \\ Tulane Ave, Rm 718A New Orleans, LA 70112 \\ Blake Kovner, BS \\ Department of Neurology Louisiana State University Health Sciences Center-New Orleans 1542 \\ Tulane Ave, Rm 718A New Orleans, LA 70112 bkovne@lsuhsc.edu
}

\begin{abstract}
Cognitive Impairment (CI) is a serious complication of MS, and the domains affected are well established but new affected domains such as theory of mind are still being identified. The evidence that some disease modifying therapies (DMTs) may improve and prevent the development of CI in MS is not solid. Recent studies on the prevalence CI in MS, although not as solid as studies completed prior to DMT introduction, suggest that CI remains a problem even among people on DMTs and even at the very earliest stages of MS. Functional MRI studies and studies using diffusion tractography show that the impact of lesions on cognition depends on the particular cortical networks affected and their plasticity. Cognitive rehabilitation and Lamphetamine appear promising treatments, cholinesterase inhibitors and memantine have failed, and data on Ginkgo and exercise are limited. We need more work to understand and develop treatment for CI in MS.
\end{abstract}

\section{Keywords}

multiple sclerosis (MS); cognitive impairment; assessment; neuropsychological tests; processing speed; memory; disease modifying therapies (DMTs); interferon; amphetamine; cholinesterase inhibitors; exercise; yoga; symptomatic treatment; cognitive rehabilitation; complementary and alternative treatments; functional connectivity; MRI; fMRI; lesion load; theory of mind; imaging correlates of ms; domains of impairment; prevalence; incidence; cortical pathology

\section{Introduction}

Cognitive Impairment (CI) is one of the most devastating complications of multiple sclerosis (MS). More than 30 reviews on the subject have been published in the last year. Particularly useful recent reviews include the work by Benedict et al [1] and Julian et al [2]. In this review we will try to include new developments not covered in these previous reviews with the objectives of determining the prevalence and incidence of CI in MS and in particular determining what the current rates are after the introduction of Disease Modifying Therapies

Corresponding author jlover@1suhsc.edu (p) 5044309932 (f) 5043240162.

Disclosure J. Lovera: EMD Serono and TEVA (consultant); B. Kovner: none. 
(DMTs), what information we have on the impact of DMTs on CI, review some novel developments in imaging correlates of $\mathrm{CI}$ and review pharmacological interventions not covered in previous reviews.

\section{Affected domains}

The domains frequently impaired in people with MS are information processing speed, working memory, verbal and visual memory, verbal fluency and executive functions being commonly impaired [3]. Two batteries have been validated and gained widespread acceptance in the assessment of CI in MS: Rao's Brief Repeatable Battery (BRB) [3] and the Minimal Assessment of Cognitive Function in MS MACFIMS [4]. A recently identified impaired domain is Theory of Mind (ToM); TOM is the ability to deduce other peoples' thoughts based on verbal and non-verbal cues. Similarly, empathy is the ability to deduce other peoples' emotions based on verbal and non-verbal cues. Banati et al recently identified ToM as another frequently impaired domain in MS [5] and functional MRI (fMRI) studies suggesting reorganization of the cortical networks responsible for ToM tasks in subjects with MS [6]. After several studies we understand that CI in MS has a pattern of impairment akin to subcortical diseases such as small vessel disease associated with aging; however, we are still identifying new impaired domains such as ToM.

\section{Prevalence, incidence and impact of $\mathrm{Cl}$ in untreated populations}

Prevalence rates of CI in MS of forty to sixty percent, derived from the studies by Rao [3] and Amato [7] respectively are frequently cited. Amato et al. have followed a cohort of MS patients recruited prior to the introduction of DMT and have followed them for more than ten years providing us with an approximate estimate of incidence rate of CI. In the study by Amato et al., the frequency of $\mathrm{CI}$ increased from $26 \%$ at baseline to $56 \%$ at the follow-up assessment ten years later [7]. Both the studies by Rao [3] and Amato [7] show that people with MS and CI are socially isolated and frequently unemployed. Morrow et al followed a cohort of 97 employed subjects with MS for 41+/-18 months with detailed neuropsychological testing and current employment determinations. They found that the Symbol Digit Modality Test (SDMT) and the California Verbal Learning Test-II-total recall both accounted for the most variance in employment and that a decrease of 2.0 points on the CVLTII-Total recall raw score and/or of 4.0 on the SDMT raw scores was associated with loss of employment [8]. D'Orio et al found, in a retrospective review of 81 patients with MS, that the occurrence of self-reported falls was increased with higher levels of impairment on verbal memory [9]. Taken together, this information indicates that without treatment CI in people with MS becomes significantly more frequent and results in a decrease in independence and quality of life.

\section{Effect of DMT on incidence of $\mathrm{Cl}$}

The prevalence and incidence estimates derived from the studies by Rao [3] and Amato [7] predate the introduction of DMTs. Unfortunately, although DMTs have been available for more than sixteen years, it is still unclear what the prevalence and incidence rates are in people with MS who have been on DMTs for most of the time since their diagnosis of MS 
and what impact the DMTs have in preventing the development of CI. Table 1 summarizes the reviewed information on DMTs and cognition.

\section{Interferon B and Glatiramer acetate (GA)}

Glatiramer acetate and various formulations of interferon B have been the main disease modifying therapies for more than ten years and they have a well established record of reducing relapses and being very safe. The Phase III trial in relapsing remitting MS of weekly intramuscular interferon B-1a included the most detailed neuropsychological assessment of the effect of a DMT in a randomized placebo controlled trial and it is a shame that other DMT trials that have followed have not made any effort to either match or improve on the methods of this landmark study. This Phase III study evaluated in detail the cognitive performance in 166 subjects that participated in the trial. It showed significant differences between the treated and placebo groups of approximately 0.4 standard deviations above the mean in the composite outcome and 1.2 standard deviations in the set of tests assessing visuo-spatial abilities and executive function [10]. Both the placebo and treated groups improved on their scores, although there was a larger improvement on the treated group and the difference in improvement was evident even at the six month evaluation. Although these results are a bit difficult to interpret because of the improvement seen also on the placebo group they suggest a beneficial effect from reduced inflammation after starting the interferon. This reduced inflammation may result in a better ability to improve on cognitive test performance, suggesting improved function of the cortical-subcortical networks responsible for learning and strategy generation after the start of the interferon. A second outcome from this study was the difference in progression of CI. The percent of patients with deterioration in their cognitive performance on the PASAT was $17 \%$ on interferon B-1a and $29 \%$ on placebo but this difference was not statistically significant [10]. Seeing an effect on the progression of CI on the PASAT is difficult because the practice effect that results in improved performance on the test will counteract the effect of the disease itself. So we have Class I evidence that treatment with intramuscular interferon B-1a results in larger improvement on cognitive performance than placebo but we have no solid evidence so far that interferon B-1a alters the rate of progression of CI in people with MS.

Improvement in delayed recall from years two to year four after randomization was seen in a small subset of 30 patients that participated in the original trial with interferon B-1b but this study did not include cognitive evaluation at the start of the study [11]. In the NorthAmerican trial of interferon B-1b for Secondary Progressive MS, interferon B-1b had no effect over a three year period of administration on the BRB [12]. Neuropsychological testing in the Phase III trial with (GA) did not show significant differences between placebo and GA groups [13]. No data on cognition are available from the three times a week subcutaneous interferon B-1a trials or any of the more recent head to head studies comparing interferon B and glatiramer acetate. It is unfortunate that so far so little data have been generated on the effect of these other DMTs on cognitive impairment in MS; the data with interferon B-1b supports the findings seen with the more robust study done with intramuscular interferon B-1a. 


\section{Natalizumab and Fingolimod}

Natalizumab and Fingolimod are newer disease modifying therapies approved for MS. The phase III studies AFFIRM [14] and SENTINEL [15] with Natalizumab did not include cognitive assessment beyond the PASAT; in the AFFIRM study, 7\% of patients on Natalizumab experienced sustained worsening performance on the PASAT versus $12 \%$ on placebo which was statistically significant. In the SENTINEL study no difference was seen on sustained worsening on the PASAT between subjects on weekly interferon-B-1a alone and those on the combination of interferon and Natalizumab [16]. Uncontrolled studies show significant improvements in cognitive function after starting Natalizumab but these studies lack of adequate control groups [17-19]. Little is known about the effects on cognition of Fingolimod; although both Phase III studies with this drug included the MS Functional Composite none of them report the changes in the PASAT component [20, 21].

Together, the results of these limited placebo controlled data with DMTs suggest that two different phenomena occur. First, there may is actual improvement of cognitive function that occurs following the start of some DMTs. This improvement is best documented in the case of intramuscular interferon B-1a and it is most prominent in visuospatial tasks, attention and executive functions and less robust in delayed recall tasks. Understanding what this improvement correlates to is difficult as after repeated testing people, even those on placebo, show significant improvement as they learn how to execute the cognitive tests better. It is tempting to hypothesize that some of this improvement after starting interferon B-1a and possibly interferon B-1b and Natalizumab results from better function in widely distributed networks as a result of a reduction in the inflammatory milieu that facilitate practice effects. This "improved practice" dominates analyses that focus on mean group performances with short term follow-up. A second type of effect is captured by analyses that focus on sustained worsening on a particular test. This sustained progression is more difficult to capture than short term improvement because it is not common, requires a longer period of follow-up to manifest and is masked by practice effects.

In these authors' opinion it is a major oversight that CI has been neglected as a relevant outcome in trials with DMTs and that after more than ten years following the introduction of Interferon B and glatiramer we still can't say for sure that they have any impact on the progression of CI in MS. It is reassuring to know that interferon B-1a has an effect on improvement in cognitive performance greater than what is seen with placebo but it is not clear that this is a clinically meaningful outcome.

\section{Incidence and prevalence of $\mathrm{Cl}$ in samples of subjects with MS treated with DMT}

Since we lack data from randomized placebo controlled studies other than the interferon B-1a observational studies and the long-term open-label extension of the placebo-controlled studies could provide us with some idea of what the incidence and prevalence of CI are in people that are treated with DMT. This is a key piece of information needed for counseling patients with MS in current times. Data regarding CI in samples of subjects who have been on DMT for most of their disease duration are scarce. The long term follow-up study of the landmark phase-III trial with GA assessed CI in 153 (67\%) of the original 251 subjects ten years after the start of the study. This study showed that worsening of more than 0.5 
standard deviations on the composite neuropsychological score derived from BRB occurred in $19 \%$ of subjects over ten years [22]. At the end of this study, 55\% of subjects had measurable impairment defined as a score worse than two standard deviations below the norm on a single test; or a score worse than one standard deviation below the mean on two tests, consistent with the overall minimal change from baseline in scores where $50 \%$ of subjects were impaired [22]. These criteria for impairment are less stringent than the ones used by Amato et al. Although the follow-up study with GA is reassuring when compared with the results from Amato et al, 39\% of subjects were lost to follow-up in the GA study. This fact can introduce significant bias and does not allow one to exclude a worse-case scenario where all of the subjects lost to follow-up were cognitively impaired.

A longitudinal observational study documented the changes in cognitive function after three years of starting treatment with either low or high dose interferon beta 1a three times a week (Rebif®) (44 mcg: $\mathrm{n}=236 ; 22 \mathrm{mcg}: \mathrm{n}=223$ ) at physician's discretion, follow-up was available for $318(69 \%)$ of the initial participants. Baseline impairment was similar in both groups (44 mcg: 15\%; $22 \mathrm{mcg}: 23.2 \%$ ) and the proportion of cognitively impaired subjects increased by $1.3 \%$ on the low dose group and remained stable in the high dose group (Hazard ratio $0.68,95 \%$ confidence interval [CI]: 0.480-0.972) suggesting a small but significant protective effect from the higher dose of interferon[23]. Other studies we found in our review (Duque et al [24], Brooks et al [25], Baumstarck-Barrau et al [26]., Brissart et al [27] and Glanz et al [28]) did not report the frequency of DMT use.

The data we found from either observational studies or extension of placebo-controlled studies is insufficient to give any meaningful answer to the question of a current patient with MS as to whether they can still expect a greater than $50 \%$ chance of developing $\mathrm{CI}$ after ten years now that we have DMTs available.

\section{Prevalence of $\mathrm{Cl}$ in Clinically Isolated Syndrome (CIS) and Radiologically Isolated Syndrome (RIS)}

DMT treatment nowadays is frequently started after the first symptoms occur and treatments are started frequently before a second attack occurs. People who have only had one attack are said to have CIS. Unfortunately, even at this early stage cognitive deficits are frequently present. Feuillet et al. found cognitive impairment in $57 \%(\mathrm{n}=40)$ of subjects with CIS three months after diagnosis ( $0.5 \mathrm{SD})$. The pattern of test results showing impairment was similar to the tests frequently impaired in subjects with longstanding MS [29]. Kocer found that subclinical dysfunction defined as impairment in at least one test from their battery was present in $80 \%$ of the CIS group and none of the controls [30]. Feinstein et al. found significant decline in visuospatial memory among 48 patients with CIS followed over a 4.5 years [31]. Reuter et al. showed rapid development of cognitive impairment in 24 subjects that were followed over 5 years after CIS diagnosis, where $29 \%$ were impaired at baseline and 54\% were impaired after five years [32]. These data indicate that individuals at the earliest stage of MSoften present with CI and highlight the importance of cognitive testing at the time of the first demyelinating attack both for counseling regarding vocational assessment and coping strategies and to monitor the progression of MS. The term Radiollogically Isolated Syndrome (RIS) refers to people with no symptoms or 
abnormalities on physical exam who have lesions typical for MS on MRI. Although very controversial, it is becoming accepted that some of these people have an even earlier stage of MS than CIS. In this situation, detailed cognitive testing, as shown by the recent studies by Lebrun [33] and Amato [34], is very useful as the pattern and frequency of impairment was comparable between subjects RIS and subjects with MS [33] and significant proportion of people with RIS (28\%) fit impairment criteria [34]. If people with RIS show signs of CI, they should not be labeled as RIS and it follows that the definition of RIS needs to be modified to: people with "no signs, symptoms, or abnormalities on physical and/or cognitive exams. This way, there is a clear difference between RIS and CIS, making the diagnostic step from RIS to CIS indicative of progressing impairment. Whether the occurrence of CI in subjects with RIS predicts the occurrence of future attacks is an important unanswered question.

\section{Imaging correlates}

Advancements in MRI technology over the last twenty-six years have enhanced tremendously our understanding of MS by providing a non-invasive means of assessment of deviations from the standard, healthy brain as well as changes in the brain over time. Overall atrophy, and in particular central atrophy-meaning atrophy of the thalamus which is reflected in the size of the third ventricle- are highly correlated with CI in MS [1].Cortical grey matter atrophy is also an important predictor of CI and although cortical atrophy and third ventricular size are highly correlated, both of them are still significant independent predictors of CI [1]. Benedict et al reviewed this interesting topic extensively[1] and as they point out, it is a well established paradox that lesion loads are not correlated with CI. Recent work by Mesaros et al [35] using diffusion tensor imaging tractography suggests that part of the lesion load paradox can be because damage to a single WM tract can affect different cognitive domains and a single cognitive domain may be affected after damage of several different WM tracts. In their study with 82 subjects with MS they were able to classify correctly $76.8 \%$ of the subjects as either cognitively impaired or not, using a novel statistical technique, the random forest strategy. Severity of fiber damage as measured by DTI in lesions in the cingulum, superior cerebellar peduncles and uncinate fasciculus were the main MRI variables driving the classification strategy [35].

Interesting new developments using fMRI have highlighted an imbalance in people with MS in the function of two opposing cortical networks the default network that is active at rest and the control network that is engaged during most cognitive tasks. Hawellek et al have examined the relationship between these two networks and CI in sixteen subjects with MS. They found that Subjects with MS with higher CI have higher connectivity in the default mode network and decreased activity in the control network. Similar findings are seen in normal aging; this finding in MS suggests that gradual but incomplete decline in the function of white matter connections and the plastic response of the brain to this decline underlies CI in MS. As the brain adapts to decline in white matter connections it makes cognitive representations such as receptive fields less specific and more broadly tuned. People with MS may have difficulties switching off their default network and turning on their control network to engage the necessary cognitive resources to sustain attention to the tasks and process information rapidly and effectively [36]. 


\section{Symptomatic treatments}

Since DMTs may not alter CI course completely and since CI may be already present at the time of diagnosis, we still need treatments that improve cognitive performance even if they do not prevent the development of CI. There has been some slow progress in this area but still much more work is needed. Table 2 summarizes the results of our review of the literature.

\section{Cognitive rehabilitation}

It may be possible to improve cognitive performance by learning strategies to use the available cognitive resources more efficiently or by doing repeated cognitive exercises that may result in a training effect. The fact that people on placebo have significant improvement on their scores on cognitive tests indicates that such practice effects really occur and that "cognitive exercise" could result in some degree of improvement similar to what physical exercise can do for improving strength.

Studies assessing the effects of "cognitive exercise" are starting to appear. A smaller study comparing subjects assigned to a six week cognitive intervention using the RehaCom software $(n=11)$, placebo $(n=14)$ or no treatment $(n=15)$ found significant improvements in executive functioning and verbal learning after six weeks of training. The verbal learning effect was sustained up to a year [37]. Chiaravalloti et al studied the approach of teaching a specific cognitive strategy showing that the training in the story memory technique did not improve verbal learning in subjects assigned to treatment $(n=14)$ vs. those assigned to placebo $(n=15)$ but a subgroup analysis did, however, show significantly improved verbal learning in the subgroup with verbal learning impairment at baseline [38]. Stuifbergen et al [39] recently reported a randomized trial comparing a comprehensive computerized cognitive rehabilitation strategy with waiting list control. They found that the rehabilitation strategy resulted in improved verbal learning, increased frequency of use of compensatory strategies and improved family member reports of the subject's cognitive function.

These early studies suggest that cognitive rehabilitation approaches can work. Much more work is needed to find the optimal therapy and determine the optimal length and intensity of administration. Further work is needed to understand how cognitive rehabilitation employs inherent neuroplasticity in a therapeutic context and whether there are ways to enhance this effect.

\section{Stimulants}

Stimulants are by definition a class of drug that yields a net increase in physical or cognitive performance. They have been shown to enhance both physical endurance and strength as they increase metabolism and intercellular concentrations of dopamine. The effects of stimulants have been tested on various facets of concentration and memory in healthy people where they are known to improve various aspects of cognitive function thus the hypothesis that they would have similar effects in people with CI from MS seems logical.

Harel et al compared the difference on the chance on the PASAT between subjects randomized to either a single dose of methyphenidate or placebo. Treatment with 
methylphenidate resulted in a significantly higher improvement on the PASAT than treatment with placebo [40]. Amphetamine is known to improve attention in ADHD and the L-enantiomer of amphetamine may have less side effects than d-amphetamine. Benedict et al [41] examined three doses (15mg, 30mg and 45mg) of the L-amphetamine and placebo on their effects on processing speed and memory in subjects with MS demonstrating cognitive impairment. Benedict et al used a cross-over design where subjects were assigned to four posible ascending sequences of single doses of $15 \mathrm{mg}$, 30mg, $45 \mathrm{mg}$ dose of L-amphetamine with placebo included after one of the amphetamine doses. The subject's performance on a neuropsychological test battery was assessed two hours after the dose to exploit peak serum levels of the study drug. After a washout period of one week the next ascending dose and its subsequent testing session were administered. L-amphetamine had no significant effect on episodic memory while processing speed and working memory improved significantly as measured by the PASAT, SDMT and part A of the trail making test with the $30 \mathrm{mg}$ and $45 \mathrm{mg}$ doses[41]. The same group evaluated the efficacy of L-amphetamine in a randomized, placebo-controlled, double-blind parallel group trial including 151 subjects from 33 MS clinics across the US treated for a period of 29 days. The score on the Subject Global Assessment of Change (SGAC) and the Symbol Digit Modalities Test (SDMT) were the primary outcome measures. The secondary outcomes were the performance on the following tests: California Verbal Learning Test-II (CVLT-II), BVMTR (Brief Visual Memory TestRevised) and PASAT. To be eligible for the study, patients had to score worse than $1.5 \mathrm{SD}$ below the mean on the SDMT or worse than one SD below the mean on the CVLT-II Delayed Recall (CVLTII-DR) or (PASAT). L-amphetamine (30mg) significantly improved the CVLTII-DR ( $p=0.012)$, BVMTR-DR ( $p<0.01)$ and BVMTR-TL scores $(p=0.041)$ but not the scores on the primary outcomes [42]. A subgroup analysis of this data showed that most of the improvement in memory function occurred among subjects with baseline memory impairment [42].

Modafinil is a stimulant that differs from amphetamine and methylphenidate in its mechanism of action as it does not work at the level of dopamine reuptake. In an open-label trial with treatment masked from evaluators, Wliken et al found that the group randomized to treatment with modafinil plus interferon B-1a $(n=23)$ performed significantly better than the group randomized to only interferon B-1a on several attention measures (Auditory Consonant Trigrams, digit span and Letter-Number Sequencing Subtest) and on the Lexical Fluency Task WAIS-III[46]. A more recent larger randomized placebo controlled trial of modafinil for fatigue [47] with 121 subjects failed to show a significant improvement on fatigue, however, the treated group had statistically significant higher improvement on the SDMT than the placebo group while the reverse occurred on the PASAT[47].

While these results are promising, the results of the trial of L-amphetamine needs to be replicated in a study that has verbal and non-verbal memory as its primary outcome and the results with methyl-phenidate need to be assessed over a longer follow-up period.

\section{Cholinesterase Inhibitors}

Acetylcholine is a neurotransmitter that facilitates memory and information processing. Several studies have indicated that decreased levels of acetylcholine in the brain are 
associated with an increase of CI in several neurodegenerative diseases including

Alzheimer's disease (AD) and Lewy Body Dementia and there is some evidence that there is a cholinergic deficit in patients with MS and this could underlie some of the CI in MS Thus it is logical to think that cholinesterase inhibitors could improve cognitive function in MS. a. In a small sample of subjects a single dose of Rivastigmine $(n=16)$ was compared to placebo $(n=16)$ and resulted in faster processing speed in a modified version of the PASAT test that can be administered while in the MRI scanner. In this study MS patients had wider prefrontal activation on fMRI than matched healthy controls. The subjects with MS that were randomized to receive a single dose of Rivastigmine showed greater improvement in their processing speed compared to the placebo group of MS subjects. After receiving Rivastigmine, the subjects with MS showed a pattern similar to that of healthy controls while the subjects with MS that received placebo still had wide prefrontal activation [43]. Treatment with Donepezil, another cholinesterase inhibitor, at $10 \mathrm{mg}$ a day for 24 weeks, had a positive effect on verbal learning, as measured with the Selective Reminding Test (SRT), in a small pilot study with 69 subjects with MS who scored 0.5 SD or more below the mean on the Rey Auditory Verbal Learning Test [44]. Unfortunately these results were not replicated in a larger multicenter trial following the same paradigm [45]. Thus, so far the results from clinical trials with cholinesterase inhibitors have been mixed with positive effects seen in small trials that were not replicated in a large, adequately powered placebocontrolled trial and thus at this time there is insufficient evidence to recommend their use. However, it is still possible that cholinesterase inhibitors have a beneficial effect in a subset of patients with $\mathrm{CI}$ and on a cognitive outcome that has not been studied so clinical research with them should not be abandoned.

\section{Memantine}

Excessive activation of the NMDA glutamate receptor is known to occur in AD and it is thought to be responsible for neuronal dysfunction responsible for a portion of the cognitive impairment that occurs in AD. There is evidence in MS of excessive glutamate concentration as well as abnormal expression of the transporters responsible for its reuptake. Memantine is a use-dependent NMDA receptor that does not have the side effects of nonselective NMDA antagonists but is still able to antagonize NMDA in situations where there is excessive glutamate release. Memantine is effective at improving cognition in patients with severe AD and since glutamate is elevate in MS it seemed plausible that Memantine could be effective at improving CI in MS. Villoslada et al had to suspend early their study of Memantine for CI in MS because of intolerable side effects[48]. We conducted a randomized placebo controlled multicenter trial with comparing treatment with memantine $(n=54)$ vs placebo $(n=60)$ in subjects with MS who performed worse than 0.5 SD below the mean on the PASAT or the CVLT-II. In our study we used a slow titration phase and this resulted in lower discontinuation rates when compared to Villoslada et al but our subjects treated with memantine still had a higher frequency of adverse events than the placebo group particularly vague neurological complains. In our study we saw no improvement in any of the cognitive tests in the battery which included the PASAT, CVLT-II, Stroop, SDMT, COWAT and DKEFS[49]. Thus memantine does not appear to be effective for CI and it may actually be harmful to people with MS. 
Ginkgo biloba-Ginkgo bilobahas unique compounds that antagonize platelet activating factor (PAF). PAF is both a neurotransmitter and an inflammatory mediator and its known to be elevated in MS. PAF works in glutaminergic synapses by serving as a retrograde transmitter that enhances the release of glutamate on the presynaptic terminal after repeated activation of the synapse. There have been several trials in AD studying the effects of Ginkgo biloba extract with the earlier trials suggesting a beneficial effect that was not reproduced in more modern trials. Since there is inflammation in MS and excessive glutamate release we hypothesized that treatment with Ginkgo biloba extract could improve cognitive performance in people with MS. We conducted a pilot randomized placebo controlled trial with Ginkgo biloba $120 \mathrm{mg}$ twice a day for 3 months where we found that subjects with MS treated with Ginkgo $(n=20)$ improved significantly more on the Stroop test than subjects treated with placebo $(n=18)$ [50]; results of our larger randomized, placebocontrolled multicenter trial with 110 subjects were presented at the 2011 American Academy of Neurology annual meeting and are in press in Neurology. The trial results were essentially negative with no effect seen in any of the cognitive outcomes [51].

Yoga and excercise-Daily physical activity has been shown to have protective effects in patients with other neurological conditions. Studies of effects of physical exercise on cognition in people with MS so far are limited. In a cross-sectional using the data from a pilot study assessing a rehabilitation intervention, Motl et al showed that there was a positive correlation between amount of daily physical exercise and processing speed but not learning and memory[52]. Yoga and aerobic exercise were evaluated in subjects with MS ( $n=69)$ in a RPCT study where subjects were randomized to yoga, exercise or a waiting list control in combination with their regular MS DMTs. Neither active treatment showed evidence of improvement in measures of attention or alertness but both active interventions improved fatigue[53]; this study did not include some yoga poses traditionally thought to improve attention and concentration traditionally thought to improve attention and concentration such as: "Flying Bird", "Standing or sitting forward bend", "Star", "Child", "Salaam", "Hare", "cat/cow" combination pose, "Savasana" corpse, "Crocodile"; "Cobra/Sphinx" combination, "Supine knee squeeze" and "Warrior/Eagle" combinations. Further research should be done on the efficacy of a combined physical and cognitive rehabilitation program including yoga and mindfulness meditation in the battery.

\section{Conclusion and future directions}

Cognitive impairment is a serious complication of MS. Intramuscular interferon B-1a improves cognitive function over the short term more than placebo and Natalizumab significantly reduces the development of sustained worsening on the PASAT. The long term effects of DMTs on cognition are uncertain. Although we hope that earlier diagnosis and treatment of MS prevents the development of CI it appears some degree of CI is present in many people with MS immediately after their initial symptoms and may be present even before symptoms develop. The pattern of cognitive dysfunction is well established but recently difficulties with theory of mind have been added to the domains frequently impaired in MS. Imaging studies continue to find interesting correlates of cognitive impairment in MS and recent studies assessing functional connectivity show that $\mathrm{CI}$ in MS 
shares an underlying pathophysiology with other processes that affect cognition such as aging. Pharmacologic as well as complementary and alternative symptomatic treatments for CI remain limited with L-amphetamine being the most promising. Cognitive rehabilitation strategies, as well as physical exercise interventions, are also showing promising results. Future studies should attempt to combine information from MRI and cognitive testing to select the best pharmacologic intervention and also look at combinations of stimulants and cognitive rehabilitation. Well designed studies to determine the impact of DMTs on cognition are greatly needed and it is unfortunate that Phase III studies of new DMTs neglect cognition as part of their secondary outcomes. Well designed long-term longitudinal observational studies are also needed but strategies to prevent loss to follow-up are critical to ensure that their results are useful.

\section{References}

1. Benedict RH, Zivadinov R. Risk factors for and management of cognitive dysfunction in multiple sclerosis. Nat Rev Neurol. 2011; 7(6):332-342. [PubMed: 21556031] Important recent review on CI in MS.

2. Julian LJ. Cognitive functioning in multiple sclerosis. Neurol Clin. 2011; 29(2):507-525. [PubMed: 21439456] Important recent review on CI in MS.

3. Rao SM, Leo GJ, Bernardin L, Unverzagt F. Cognitive dysfunction in multiple sclerosis. I. Frequency, patterns, and prediction. Neurology. 1991; 41(5):685-691. [PubMed: 2027484]

4. Benedict RH, Fischer JS, Archibald CJ, et al. Minimal neuropsychological assessment of MS patients: a consensus approach. Clin Neuropsychol. 2002; 16(3):381-397. [PubMed: 12607150]

5. Banati M, Sandor J, Mike A, et al. Social cognition and Theory of Mind in patients with relapsingremitting multiple sclerosis. Eur J Neurol. 2009; 17(3):426-433. [PubMed: 19922457]

6. Jehna M, Langkammer C, Wallner-Blazek M, et al. Cognitively preserved MS patients demonstrate functional differences in processing neutral and emotional faces. Brain Imaging Behav. 2011; 5(4): 241-251. [PubMed: 21656213] Interesting new findings on the changes in cortical networks responsible theory of mind processes in patients with MS.

7. Amato MP, Ponziani G, Siracusa G, Sorbi S. Cognitive dysfunction in early-onset multiple sclerosis: a reappraisal after 10 years. Arch Neurol. 2001; 58(10):1602-1606. [PubMed: 11594918]

8. Morrow SA, Drake A, Zivadinov R, et al. Predicting loss of employment over three years in multiple sclerosis: clinically meaningful cognitive decline. Clin Neuropsychol. 2010; 24(7):11311145. [PubMed: 20830649] This important paper helps determine a level of change in cognitive tests that has important implications for employability and will be useful in the design of clinical trials.

9. D'Orio VL, Foley FW, Armentano F, et al. Cognitive and motor functioning in patients with multiple sclerosis: Neuropsychological predictors of walking speed and falls. J Neurol Sci. 2012; 316(1-2):42-46. [PubMed: 22353853]

10. Fischer JS, Priore RL, Jacobs LD, et al. Neuropsychological effects of interferon beta-1a in relapsing multiple sclerosis. Multiple Sclerosis Collaborative Research Group. Ann Neurol. 2000; 48(6):885-892. [PubMed: 11117545]

11. Pliskin NH, Hamer DP, Goldstein DS, et al. Improved delayed visual reproduction test performance in multiple sclerosis patients receiving interferon beta-1b. Neurology. 1996; 47(6): 1463-1468. [PubMed: 8960728]

12. Panitch H, Miller A, Paty D, Weinshenker B. Interferon beta-1b in secondary progressive MS: results from a 3-year controlled study. Neurology. 2004; 63(10):1788-1795. [PubMed: 15557491]

13. Weinstein A, Schwid SR, Schiffer RB, et al. Neuropsychologic status in multiple sclerosis after treatment with glatiramer. Arch Neurol. 1999; 56(3):319-324. [PubMed: 10190822]

14. Polman CH, O'Connor PW, Havrdova E, et al. A randomized, placebo-controlled trial of natalizumab for relapsing multiple sclerosis. N Engl J Med. 2006; 354(9):899-910. [PubMed: 16510744] 
15. Rudick RA, Stuart WH, Calabresi PA, et al. Natalizumab plus interferon beta-1a for relapsing multiple sclerosis. N Engl J Med. 2006; 354(9):911-923. [PubMed: 16510745]

16. Weinstock-Guttman B, Galetta SL, Giovannoni G, et al. Additional efficacy endpoints from pivotal natalizumab trials in relapsing-remitting MS. J Neurol. 2011 online. Important although limited assessment of the impact of Natalizumab on CI.

17. Lang C, Reiss C, Maurer M. Natalizumab may improve cognition and mood in multiple sclerosis. Eur Neurol. 67(3):162-166. [PubMed: 22269396]

18. Mattioli F, Stampatori C, Capra R. The effect of natalizumab on cognitive function in patients with relapsing-remitting multiple sclerosis: preliminary results of a 1-year follow-up study. Neurol Sci. 32(1):83-88. [PubMed: 20872033]

19. Morrow SA, O'Connor PW, Polman CH, et al. Evaluation of the symbol digit modalities test (SDMT) and MS neuropsychological screening questionnaire (MSNQ) in natalizumab-treated MS patients over 48 weeks. Mult Scler. 16(11):1385-1392. [PubMed: 20739335]

20. Cohen JA, Barkhof F, Comi G, et al. Oral fingolimod or intramuscular interferon for relapsing multiple sclerosis. N Engl J Med. 2010; 362(5):402-415. [PubMed: 20089954]

21. Kappos L, Radue EW, O'Connor P, et al. A placebo-controlled trial of oral fingolimod in relapsing multiple sclerosis. N Engl J Med. 2010; 362(5):387-401. [PubMed: 20089952]

22. Schwid SR, Goodman AD, Weinstein A, et al. Cognitive function in relapsing multiple sclerosis: minimal changes in a 10-year clinical trial. J Neurol Sci. 2007; 255(1-2):57-63. [PubMed: 17331542]

23. Patti F, Amato MP, Bastianello S, et al. Effects of immunomodulatory treatment with subcutaneous interferon beta-1a on cognitive decline in mildly disabled patients with relapsingremitting multiple sclerosis. Mult Scler. 2009; 16(1):68-77. [PubMed: 19995846]

24. Duque B, Sepulcre J, Bejarano B, et al. Memory decline evolves independently of disease activity in MS. Mult Scler. 2008; 14(7):947-953. [PubMed: 18573817]

25. Brooks JB, Borela MC, Fragoso YD. Assessment of cognition using the Rao's Brief Repeatable Battery of Neuropsychological Tests on a group of Brazilian patients with multiple sclerosis. Arq Neuropsiquiatr. 2012; 69(6):887-891. [PubMed: 22297873]

26. Baumstarck-Barrau K, Simeoni MC, Reuter F, et al. Cognitive function and quality of life in multiple sclerosis patients: a cross-sectional study. BMC Neurol. 2011; 11:17. [PubMed: 21288343]

27. Brissart H, Morele E, Baumann C, Debouverie M. Verbal episodic memory in 426 multiple sclerosis patients: impairment in encoding, retrieval or both? Neurol Sci. 2012

28. Glanz BI, Healy BC, Hviid LE, et al. Cognitive deterioration in patients with early multiple sclerosis: a 5-year study. J Neurol Neurosurg Psychiatry. 2011; 83(1):38-43. [PubMed: 21746743]

29. Feuillet L, Reuter F, Audoin B, et al. Early cognitive impairment in patients with clinically isolated syndrome suggestive of multiple sclerosis. Mult Scler. 2007; 13(1):124-127. [PubMed: 17294621]

30. Kocer B, Unal T, Nazliel B, et al. Evaluating sub-clinical cognitive dysfunction and event-related potentials (P300) in clinically isolated syndrome. Neurol Sci. 2008; 29(6):435-444. [PubMed: 19002651]

31. Feinstein A, Kartsounis LD, Miller DH, et al. Clinically isolated lesions of the type seen in multiple sclerosis: a cognitive, psychiatric, and MRI follow up study. J Neurol Neurosurg Psychiatry. 1992; 55(10):869-876. [PubMed: 1431949]

32. Reuter F, Zaaraoui W, Crespy L, et al. Frequency of cognitive impairment dramatically increases during the first 5 years of multiple sclerosis. J Neurol Neurosurg Psychiatry. 2010; 82(10):11571159. [PubMed: 20971755]

33. Lebrun C, Blanc F, Brassat D, et al. Cognitive function in radiologically isolated syndrome. Mult Scler. 2010; 16(8):919-925. [PubMed: 20610492] This interesting study documents the presence of cognitive changes before other symptoms occur among people with incidental findings of brain lesions typical of MS.

34. Amato MP, Hakiki B, Goretti B, et al. Association of MRI metrics and cognitive impairment in radiologically isolated syndromes. Neurology. 2012; 78(5):309-314. [PubMed: 22262744] Another recent interesting study documenting CI in patients with RIS. 
35. Mesaros S, Rocca MA, Kacar K, et al. Diffusion tensor MRI tractography and cognitive impairment in multiple sclerosis. Neurology. 2012; 78(13):969-75. [PubMed: 22377806] This study clarifies the long standing paradox of why lesion burden does not correlate with CI.

36. Hawellek DJ, Hipp JF, Lewis CM, et al. Increased functional connectivity indicates the severity of cognitive impairment in multiple sclerosis. Proc Natl Acad Sci U S A. 2011; 108(47):1906619071. [PubMed: 22065778] This study documents changes in the default and control networks in patients with MS and correlates this changes with CI.

37. Fink F, Rischkau E, Butt M, et al. Efficacy of an executive function intervention programme in MS: a placebo-controlled and pseudo-randomized trial. Mult Scler. 2010; 16(9):1148-1151. [PubMed: 20621945]

38. Chiaravalloti ND, DeLuca J, Moore NB, Ricker JH. Treating learning impairments improves memory performance in multiple sclerosis: a randomized clinical trial. Mult Scler. 2005; 11(1):5868. [PubMed: 15732268]

39. Stuifbergen AK, Becker H, Perez F, et al. A randomized controlled trial of a cognitive rehabilitation intervention for persons with multiple sclerosis. Clin Rehabil. 2012 online. This study shows promising results from cognitive rehabilitation strategy.

40. Harel Y, Appleboim N, Lavie M, Achiron A. Single dose of methylphenidate improves cognitive performance in multiple sclerosis patients with impaired attention process. J Neurol Sci. 2009; 276(1-2):38-40. [PubMed: 18817930]

41. Benedict RH, Munschauer F, Zarevics P, et al. Effects of l-amphetamine sulfate on cognitive function in multiple sclerosis patients. J Neurol. 2008; 255(6):848-852. [PubMed: 18481035]

42. Sumowski JF, Chiaravalloti N, Erlanger D, et al. L-amphetamine improves memory in MS patients with objective memory impairment. Mult Scler. 2011; 17(9):1141-1145. [PubMed: 21561956]

43. Wilken JA, Sulliven C, Wallin M, et al. Treatment of Multiple Sclerosis-Related Cognitive Problems With Adjunctive Modafinil. Int J MS Care. 2008; 10:1-10.

44. Moller F, Poettgen J, Broemel F, et al. HAGIL (Hamburg Vigil Study): a randomized placebocontrolled double-blind study with modafinil for treatment of fatigue in patients with multiple sclerosis. Mult Scler. 2011; 17(8):1002-1009. [PubMed: 21561959]

45. Huolman S, Hamalainen P, Vorobyev V, et al. The effects of rivastigmine on processing speed and brain activation in patients with multiple sclerosis and subjective cognitive fatigue. Mult Scler. 2011; 17(11):1351-1361. [PubMed: 21846692]

46. Krupp LB, Christodoulou C, Melville P, et al. Donepezil improved memory in multiple sclerosis in a randomized clinical trial. Neurology. 2004; 63(9):1579-1585. [PubMed: 15534239]

47. Krupp LB, Christodoulou C, Melville P, et al. Multicenter randomized clinical trial of donepezil for memory impairment in multiple sclerosis. Neurology. 2011; 76(17):1500-1507. [PubMed: 21519001] This is an important randomized placebo controlled trial that unfortunately showed no benefit with donepezil.

48. Villoslada P, Arrondo G, Sepulcre J, et al. Memantine induces reversible neurologic impairment in patients with MS. Neurology. 2009; 72(19):1630-1633. [PubMed: 19092106]

49. Lovera JF, Frohman E, Brown TR, et al. Memantine for cognitive impairment in multiple sclerosis: a randomized placebo-controlled trial. Mult Scler. 2010; 16(6):715-723. [PubMed: 20483885] Our randomized multicenter trial with memantine also failed to show any beneficial effect.

50. Lovera J, Bagert B, Smoot K, et al. Ginkgo biloba for the improvement of cognitive performance in multiple sclerosis: a randomized, placebo-controlled trial. Mult Scler. 2007; 13(3):376-385. [PubMed: 17439907]

51. Lovera J, Howieson D, Wild K, et al. Randomized placebo controlled trial of Ginkgo biloba for cognitive impairment in multiple sclerosis. Neurology. 2011; (Supp 4):A546.

52. Motl RW, Gappmaier E, Nelson K, Benedict RH. Physical activity and cognitive function in multiple sclerosis. J Sport Exerc Psychol. 2011; 33(5):734-741. [PubMed: 21984644]

53. Oken BS, Kishiyama S, Zajdel D, et al. Randomized controlled trial of yoga and exercise in multiple sclerosis. Neurology. 2004; 62(11):2058-2064. [PubMed: 15184614] 


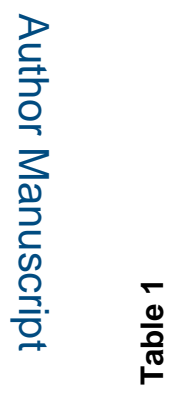

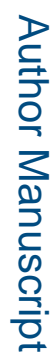

\begin{tabular}{|c|c|c|c|c|c|c|c|c|c|c|}
\hline 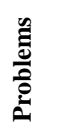 & & & & & 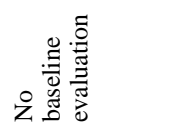 & & & & & $\begin{array}{l}\text { 㺃 } \\
\text { O } \\
\text { z }\end{array}$ \\
\hline 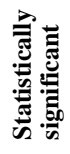 & $\stackrel{\mathscr{3}}{-\infty}$ & $\stackrel{2}{z}$ & ż & z & 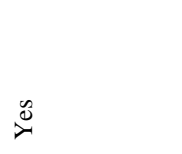 & z & $\dot{z}$ & $\dot{z}$ & $\stackrel{\infty}{=}$ & \\
\hline 흘 & 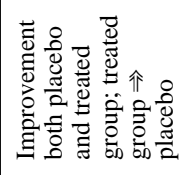 & 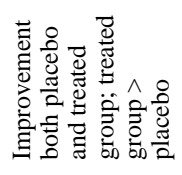 & 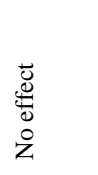 & 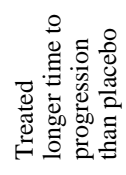 & 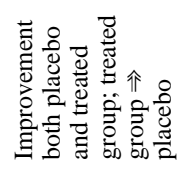 & 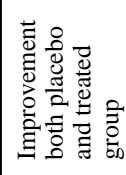 & 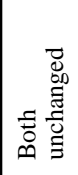 & 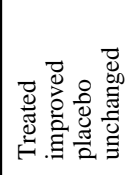 & 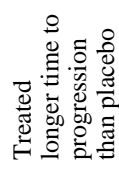 & \\
\hline 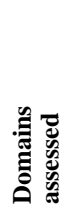 & 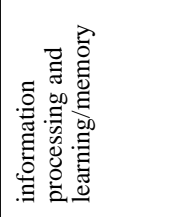 & 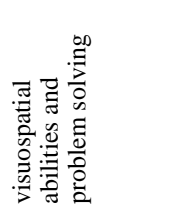 & 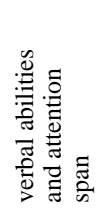 & 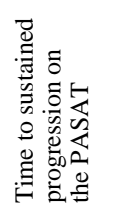 & 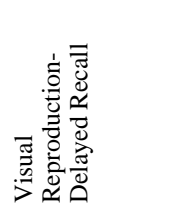 & 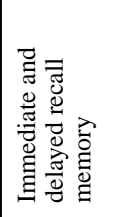 & 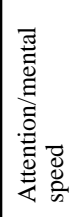 & 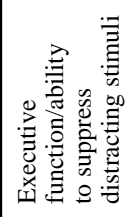 & 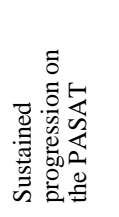 & \\
\hline 言言 & $\begin{array}{l}0 \\
\frac{n}{0} \\
3 \\
3 \\
0 \\
0\end{array}$ & & & & 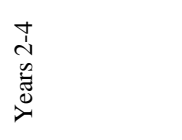 & & & & 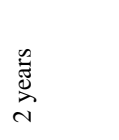 & \\
\hline z & $\stackrel{\circ}{\circ}$ & & & & ల & & & & Ĩ & \\
\hline$\sum_{i}$ & 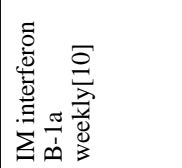 & & & & 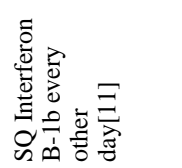 & & & & 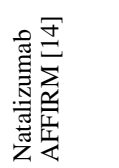 & 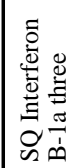 \\
\hline
\end{tabular}

Curr Neurol Neurosci Rep. Author manuscript; available in PMC 2015 September 24. 


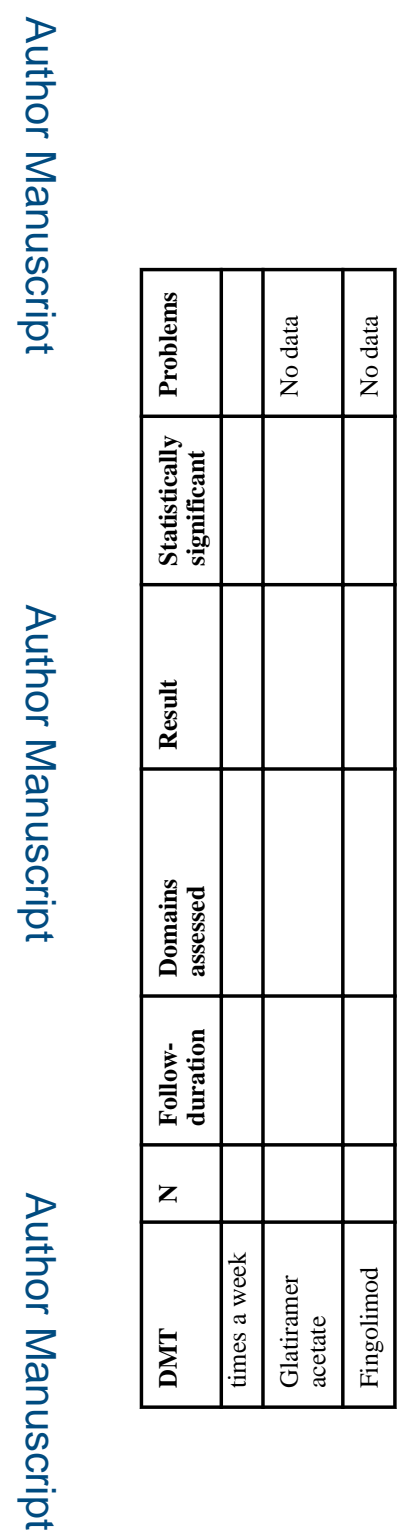




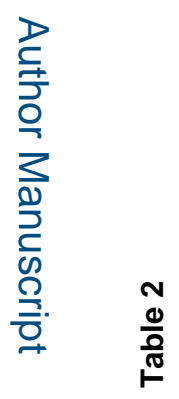

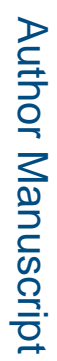

\begin{tabular}{|c|c|c|c|c|c|c|c|c|c|c|c|c|}
\hline 施 & & & & & 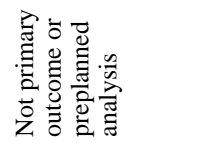 & & & 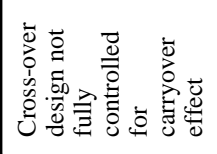 & & & 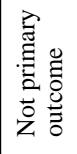 & 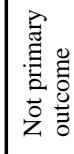 \\
\hline 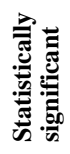 & $\overbrace{=}^{\infty}$ & $\stackrel{\infty}{\infty}$ & $\stackrel{\infty}{\nearrow}$ & $\stackrel{2}{z}$ & $\stackrel{\infty}{\check{C}}$ & & $\overbrace{\nu}^{\infty}$ & $\stackrel{\infty}{2}$ & $\underset{\nu}{\mathscr{0}}$ & & $\overbrace{\nu}^{\infty}$ & $\stackrel{\circlearrowright}{\varnothing}$ \\
\hline $\begin{array}{l}\overline{\bar{z}} \\
\stackrel{\bar{g}}{\cong}\end{array}$ & 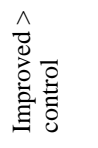 & 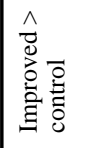 & 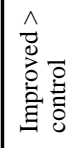 & 总 & 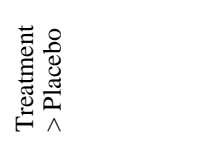 & & 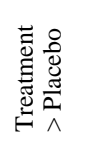 & 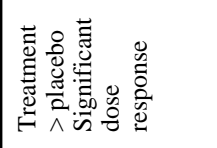 & z: & 总 & 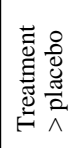 & 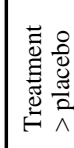 \\
\hline 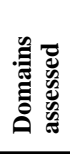 & 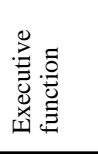 & 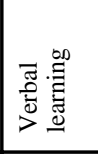 & 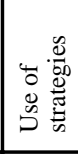 & 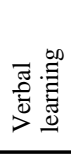 & 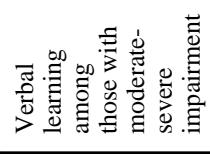 & & 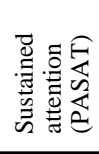 & 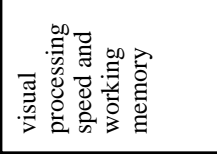 & 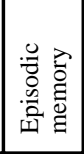 & 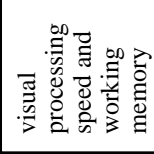 & 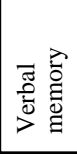 & 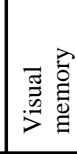 \\
\hline 施 & 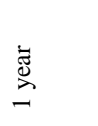 & 量 & & 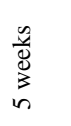 & & & $\begin{array}{l}\overline{\bar{z}} \\
\underline{\underline{z}}\end{array}$ & $\frac{n}{\vdots}$ & & 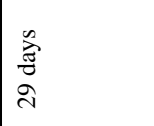 & & \\
\hline z & in & $\bar{n}$ & & ते & & & i & 2 & & $\stackrel{n}{2}$ & & \\
\hline 䓪 & 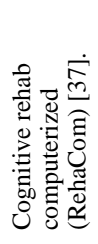 & 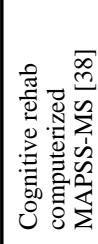 & & 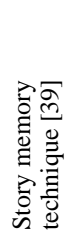 & & 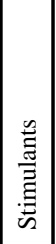 & 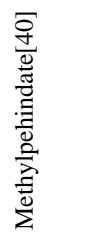 & 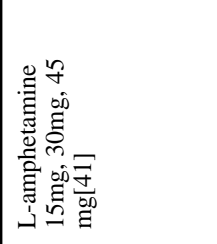 & & 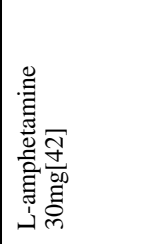 & & \\
\hline
\end{tabular}

Curr Neurol Neurosci Rep. Author manuscript; available in PMC 2015 September 24. 


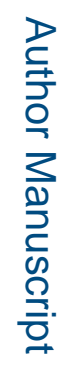

로을

\begin{tabular}{|c|c|c|c|c|c|c|c|c|c|c|c|}
\hline$\frac{n}{\frac{n}{2}}$ & 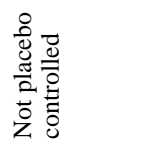 & 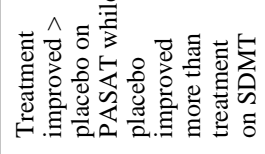 & & & & & 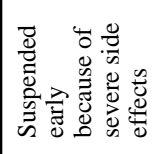 & 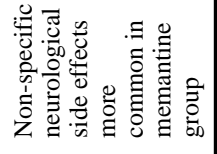 & & & \\
\hline 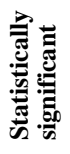 & 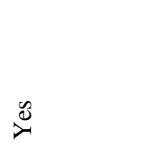 & $\lambda$ & & $\overbrace{\nearrow}^{\infty}$ & $\check{\infty}_{0}^{\infty}$ & & & & & & \\
\hline 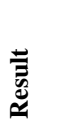 & 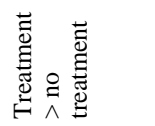 & 芠 & & 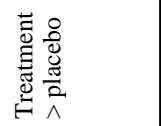 & 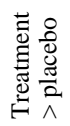 & 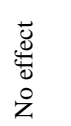 & & 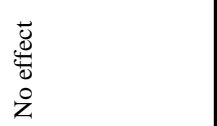 & 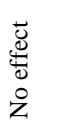 & 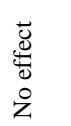 & 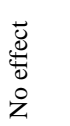 \\
\hline 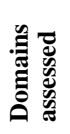 & 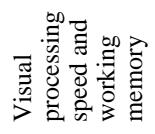 & 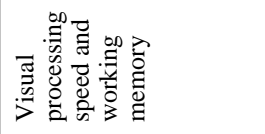 & & 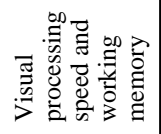 & 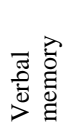 & 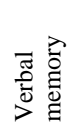 & 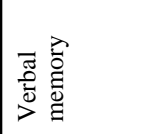 & 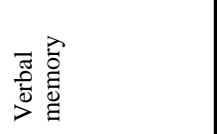 & 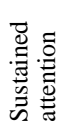 & 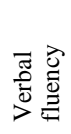 & 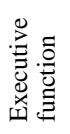 \\
\hline 旅 & $\begin{array}{l}\text { 告 } \\
\text { 音 } \\
\text { g }\end{array}$ & $\begin{array}{l}n \\
\frac{n}{0} \\
0 \\
\infty \\
\infty\end{array}$ & & 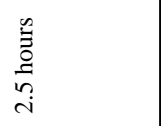 & 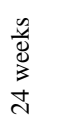 & 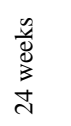 & & 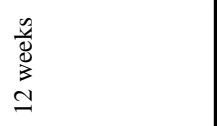 & & & \\
\hline z & 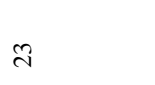 & $\bar{\Xi}$ & & $\stackrel{0}{2}$ & 8 & తి & 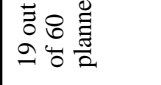 & $\stackrel{\varrho}{\exists}$ & & & \\
\hline & 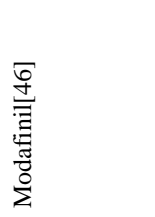 & 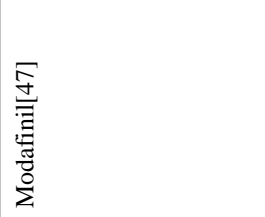 & 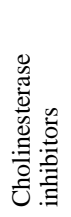 & 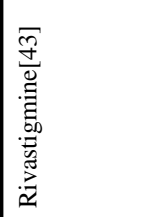 & 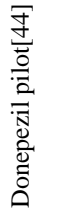 & 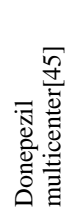 & 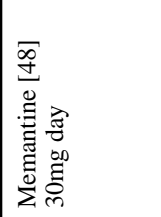 & 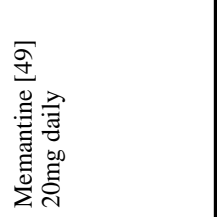 & & & \\
\hline
\end{tabular}

Curr Neurol Neurosci Rep. Author manuscript; available in PMC 2015 September 24. 


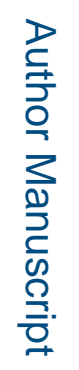

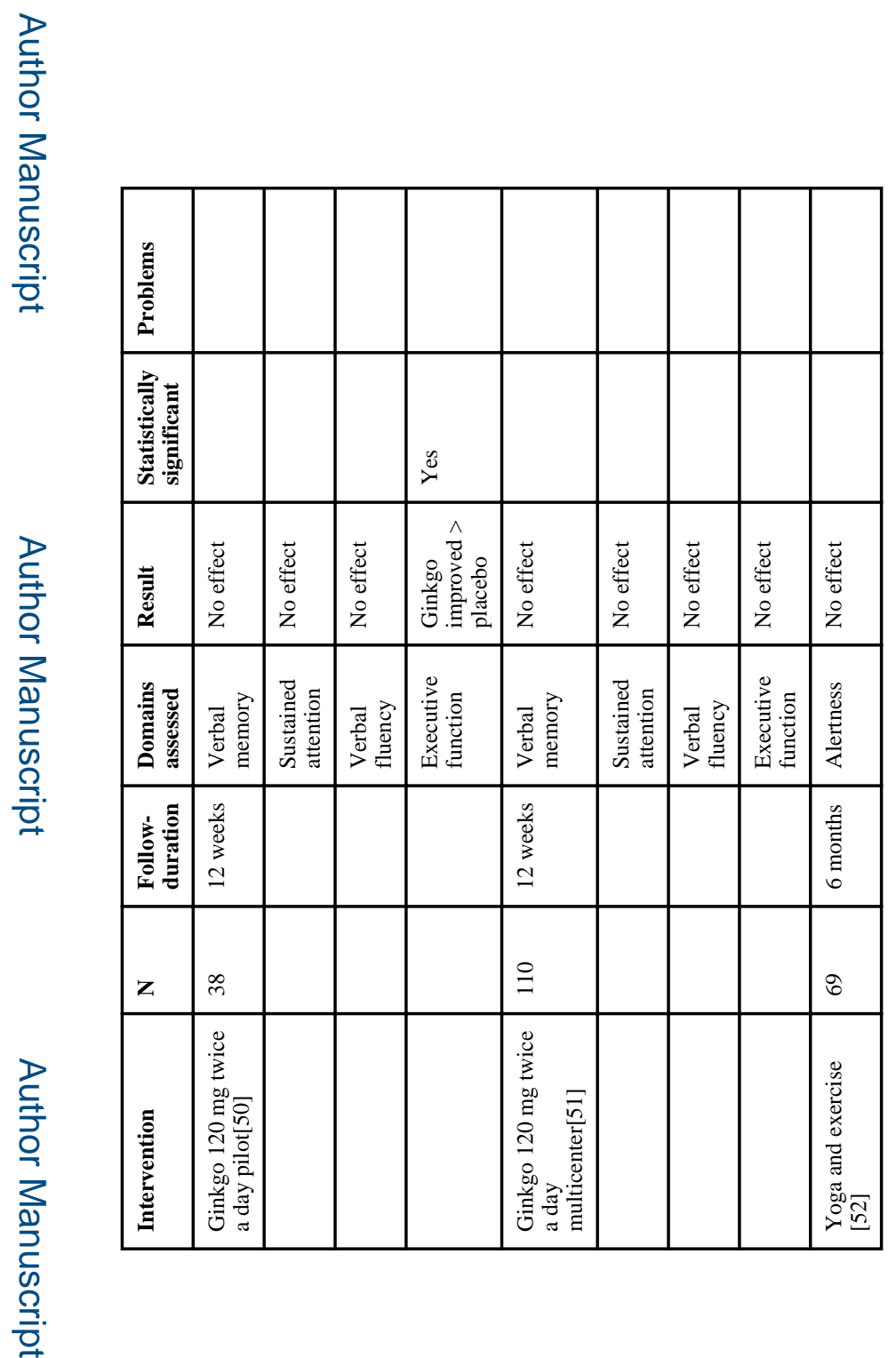

Curr Neurol Neurosci Rep. Author manuscript; available in PMC 2015 September 24. 